\title{
COMPARISON OF POINT AND SEGMENT BASED POINT CLOUD CLASSIFICATION METHOD IN URBAN SCENES
}

\author{
Erfan Hasanpour ${ }^{1, *}$, Mohammad Saadat Seresht ${ }^{1}$, Ebadat Ghanbari Parmehr ${ }^{2}$ \\ ${ }^{1}$ University of Tehran, School of Surveying and Geospatial Engineering - (erfan.hasanpoor, msaadat)@ut.ac.ir \\ ${ }^{2}$ Babol Noshirvani University of Technology, dept. of Geomatics, Faculty of Civil Engineering - parmehr@ nit.ac,ir
}

KEY WORDS: Classification, Point Cloud, Comparison of Features, Random Forest

\begin{abstract}
:
Point cloud classification is an essential requirement for effectively utilizing point cloud data acquired by different kind of sources such as Terrestrial Laser Scanning (TLS), Aerial LiDAR (Light Detection and Ranging), and Photogrammetry. Classification of point cloud is a process that points are separated into different point groups that each group has similar features. Point cloud classification can be done in three levels (point-based, segment-based, and object-based) and the choice of different level has significant impact on classification result. In this research, random forest classification method is utilized in which the pointwise and segment-wise spectral and geometric features are selected as the input of the classification. In our experiments, the results of point- and segment-based classification were compared. In addition, point-wise classification result for two different features (geometric with/without spectral features) has been compared and the results are presented. The experiments illustrated that segment based classification with both color and geometric features has the best overall accuracy of $83 \%$ especially near the object boundaries.
\end{abstract}

\section{INTRODUCTION}

3D Point clouds provide enrich spatial information for urban scenes and have been widely used for many applications. The aim of point cloud classification is separation of different points that belong to different objects.

The classification process has four key steps including neighborhood selection, feature selection and extraction, choice of classifier and classification of points based on the respective features. The neighborhood selection has always been the focus of many studies. Most of previous studies used point-base neighborhood selection that in this method neighborhood points around each point are selected and for each point and its neighborhood features are extracted. $\mathrm{K}$ nearest neighbor (KNN) and spherical neighborhood are point-base methods that in many previous studies were used. KNN are more useful than spherical neighborhood for point cloud because of point density variation. Some authors have proposed voxel or supervoxel-based neighborhoods for feature extraction. In the following, some classification researches are mentioned based on different neighborhood and classifier selections. Bremer, et al proposed a rule-based classification based on point-base neighborhood detection (Bremer et al, 2013). Ramiya et al uses supervoxelbased segmentation to segment the point cloud data first, and then uses different machine-learning algorithms to label the point cloud (Chehata et al, 2009). Luo, et al proposed a supervoxel-based classification, their method used Conditional Random Field matching to classify supervoxels (Luo, $\mathrm{H}$ et al, 2018). Sun, et al used Random Forest classifier to classify point cloud based on supervoxels (Sun, Z et al, 2018). Yang, et al utilized Region Growing algorithm for segmentation of point cloud and SVM classifier for object based classification (Yang, $\mathrm{B}$ et al, 2017).The segment-wise classification is used to over segment the point and then features are extracted for each segment. In this research point cloud is segmented based on ortho image super pixel generation. We investigated geometric features and color features. Point cloud acquired by laser scanning has intensity features but here, point clouds derived from UAV images and so intensity features don't exist. Geometric features can be more important for objects that have regular structure and color features can be more important for vegetation. Experiment results will demonstrated that combination of color geometric information is able to improve the classification results. The remainder of this paper is organized as follows: the proposed method is described in Section 2, the results are described in Section 3, and finally, conclusions follow in Section 4.

\section{PROPOSED METHOD}

The general framework of this study is given in Figure1.Two kinds of comparison have been conducted, first comparison is between point-based and segment-base feature extraction and second comparison is between choices of different features. 23 features for different type of neighborhood (point-wise and segment-wise) are extracted, and then features were used to train random forest classifier in the training stage and predict labels. In total pointcloud in 3 different condition are classified that are include: segment-base classification that both color and geometric features are used, point-base classification that both color and geometric feature are used and point-base classification that only geometric feature are used. Finally the Specificity, Sensitivity, Prevalence and overall accuracy are used to evaluate the classification performance.

* Corresponding author 


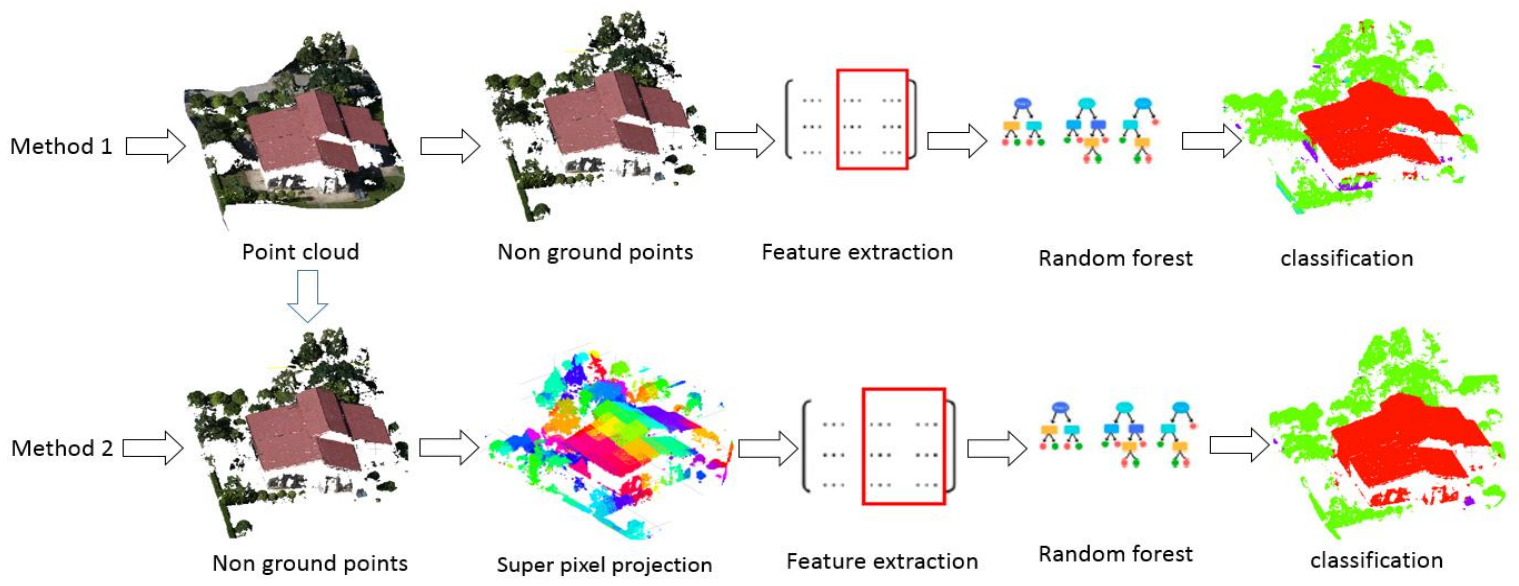

Figure 1. The general framework of the study

\subsection{Ground Filtering}

In this research, four classes including roof, tree, car and grass are non-ground points has been used. Since all of ground points have the same height, geometric features can not help for classification stage, points are seperated into ground point and non-ground to reduce computation time. Therefore, ground points were extracted by cloth simulation filtering(csf) algoritm proposed by (zhang et al, 2016) and non ground points were used for point cloud classification.
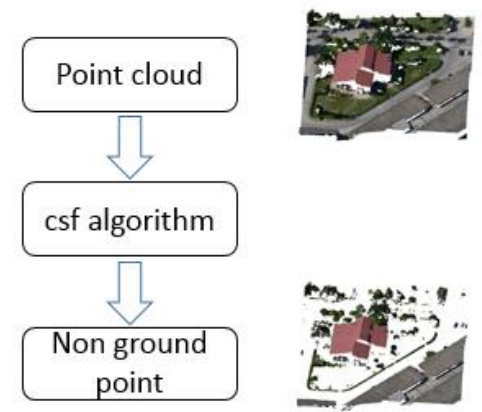

Figure 2. Ground point filtering

\subsection{Superpixel Projection}

Classification or segmentation process are based on selection of a certain neighborhood for extraction different features. Neighborhood can be selected as point-based methods like KNN for each point or segment-based methods like voxel generation. Both methods have some problems. point-based methods have too much computation that consume a lot of times. Computation speed in voxel base mthods are more than point-based methods but edge points can be lied in voxels, so superpixel method are used to separate point based on edges in ortho image and reduce the computational time. Since the first method, point cloud classification method is based on points, the super pixels is not necessary, but for the second method super pixel generation is used to over segment points .

As workflow that are shown in figure3, First, ortheimage was segmented by SLIC algorithm derived by (Radhakrishna et al,
2012) and then superpixels were porjected onto point cloud Super pixels on point cloud are as supervoxel that consider to edge points. super pixel are generated by image color features and then are projected. Segments that have points less than a certain threshold are removed. Points lie in each sgment are considerd as a certain neighborhood and for classification and feature selection step, these segments are as input.

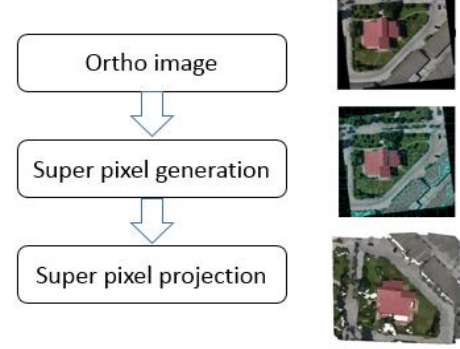

Figure 3. Super pixel generation and projection

\subsection{Feature Extraction}

After supervoxel generation, different features are extracted based on the supervoxel neighborhood. Feature selection and extraction constitute the essential part of point cloud classification, and their performance plays a decisive role in classification results (Quan Li et al, 2018).in this study we used two kind of features that are known as geometric and color features. Geometric features are mainly comprised of covariance features that are derived from normalized eigenvalues $(\lambda 1, \lambda 2$, $\lambda 3)$. Eigenvalues are useful for representation local geometric shape in a certain neighborhood and if assume eigenvalues are stored as $\lambda 1>\lambda 2>\lambda 3, \lambda 3$ is as normal vector of point in certain neighborhood. Besides covariance features, three geometric features include mean $\mathrm{z}$ value, $\mathrm{z}$ variance, and maximum $\mathrm{z}$ difference that are derived from height are used. As color features, 12 features are considered that include: mean R, G, and $\mathrm{B} ; \mathrm{R}, \mathrm{G}$, and $\mathrm{B}$ ratio; $\mathrm{R}, \mathrm{G}$, and $\mathrm{B}$ variance, and maximum $\mathrm{R}, \mathrm{G}$, and B difference (Quan Li et al, 2018).

For both of methods, we used the same features that are shown in table 1. We used two kinds of features, 11 geomatics features 
and 12 color features. Features derived by Quant Li et al (Quan Li et al, 2018).

For point-based method features were extracted for 10 neighborhood points around each point, but for segment-based method, features were extracted for points inside each super pixel.

\section{Geometric Features}

Linearity
Planarity
Sphericity
Omnivariance
Anisotropy
Eigenentropy
Sum of eigenvalues
Change of curvature
Mean $Z$
$Z$ variance
Maximum $Z$ difference

\section{Color Features}

$\left(\lambda_{1}-\lambda_{2}\right) / \lambda_{1}$
$\left(\lambda_{2}-\lambda_{3}\right) / \lambda_{1}$
$\lambda_{3} / \lambda_{1}$
$\left(\lambda_{1} \cdot \lambda_{2} \cdot \lambda_{3}\right)^{1 / 3}$
$\left(\lambda_{1}-\lambda_{3}\right) / \lambda_{1}$
$-\sum_{i=1}^{3} \lambda_{i} \cdot \ln \left(\lambda_{i}\right)$
$\lambda_{1}+\lambda_{2}+\lambda_{3}$
$\lambda_{3} /\left(\lambda_{1}+\lambda_{2}+\lambda_{3}\right)$
$\left(\sum_{i=1}^{n} Z_{i}\right) / n$
$\left(\sum_{i=1}^{n} Z_{i}-\bar{Z}\right) / n$
$Z_{\max }-Z_{\min }$
Mean R Mean G Mean B $\mathrm{R}$ ratio G ratio B ratio: $R$ variance G variance B variance

Maximum R difference Maximum $\mathrm{G}$ difference Maximum $B$ difference

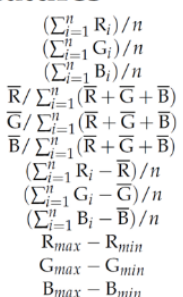

Table 1. Two types of feature sets [1]

\subsection{Classifier}

In this research, random forest has been used as classifier to classify point cloud data. Random forest is a set of decision trees that from a training set's randomly selected subsets (Breiman et al, 2001). Random forest proved that it has a good performance for point cloud classification (Chehata et al, 2009). We trained 3 random forest classifiers.

They were trained with 11 geomatics feature for point-base method, 11 geomatics features combined with 12 color feature for point base method and 11 geomatics feature combined with 12 color features for segment-base method.

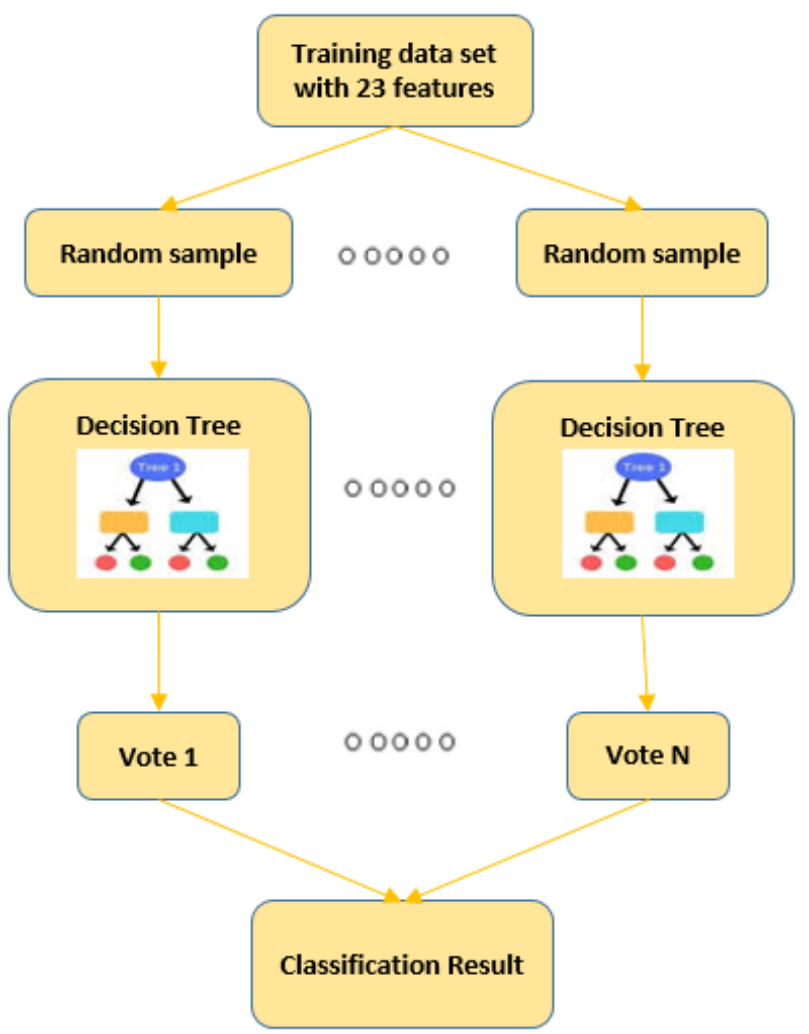

Figure 4. Random forest classification of feature vectors

\section{RESULTS}

In this study, point cloud data are derived from UAV images and acquired by phantom 4 . This data presents a typical urban scene from university of Babol. This data have 5,735,827 points that after ground filtering is include 2,783,553 points.

The random forest results for point-based method using different features have been compared. First random forest was trained for 11 geometric features along with 12 color features and then it was trained with only 11 geometric features. Results are shown in Fig 5. As shown in Fig 5, without color features, trees and roofs are mistaken as well as grass and cars. Color features are useful for objects with specified color features such as trees and vegetation.

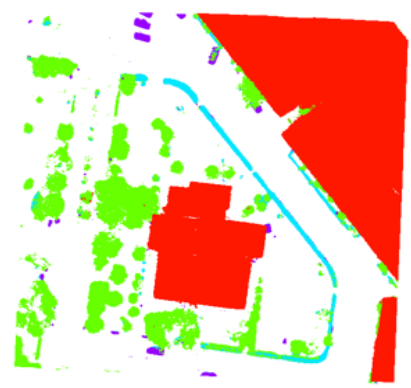

Geomatics and color features

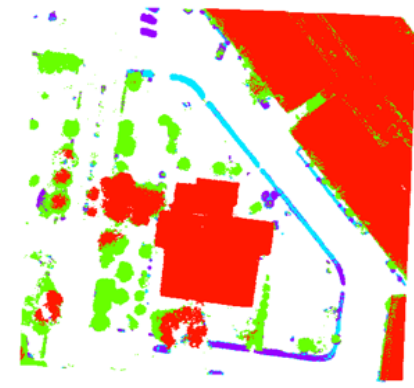

Geomatics features

Figure 5. Classification results for different features (tree $=$ green, roof $=$ red, grass $=$ blue, car $=$ purple)

Point and segment-based classification methods have been compared. The results are shown in Fig 5. The results are compared with ground truth and shown in table 2. As table2 segment-based method improve the classification result and also reduce the computation time, it has good performance for places that trees are near building. Segment-based method did not provide acceptable results for grass class and it have some problems for edge points however it has homogenous regions with lower noise for each class.

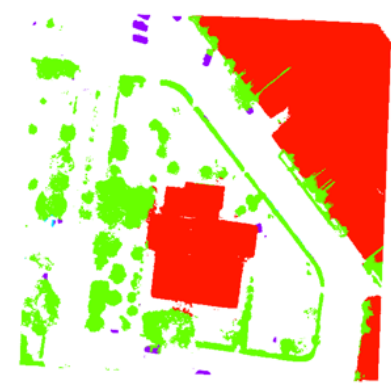

Segment-base classification

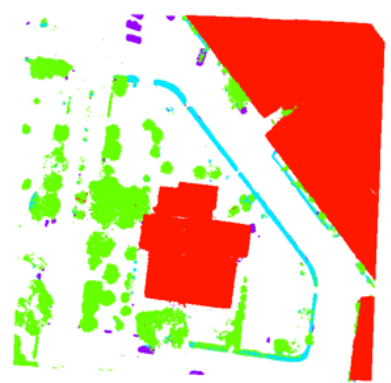

Point-base classification

Figure 6. Classification results (tree $=$ green, roof $=$ red, grass=blue, car $=$ purple) 


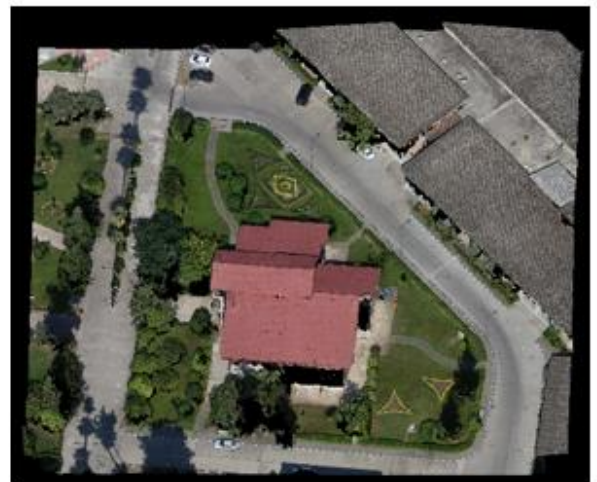

Original pointcloud

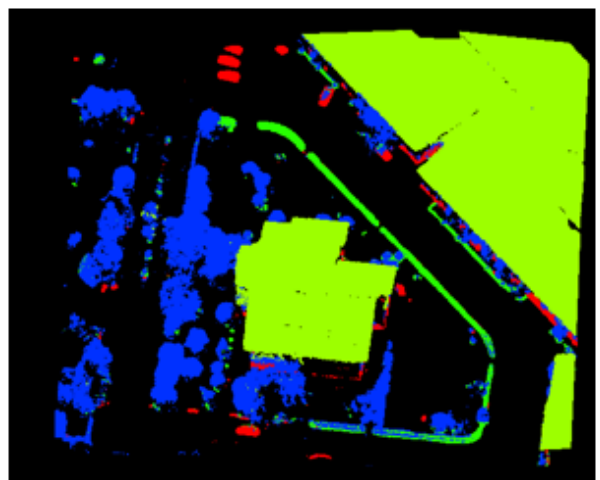

Point-based classification using both features

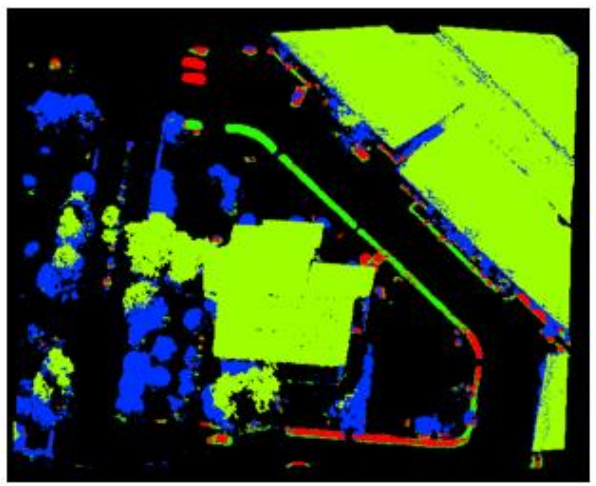

Point-based classification using geometric features

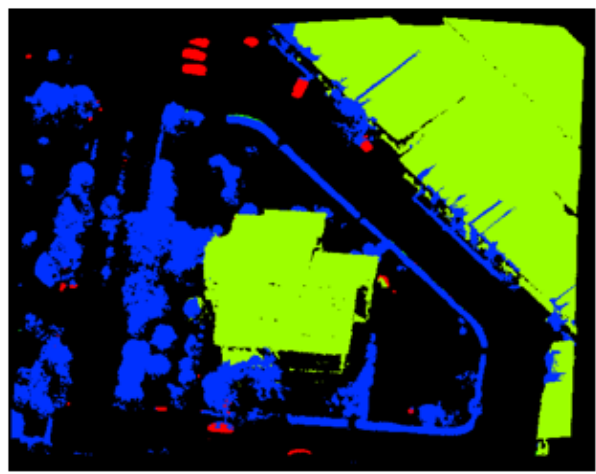

segment-based classification using both features

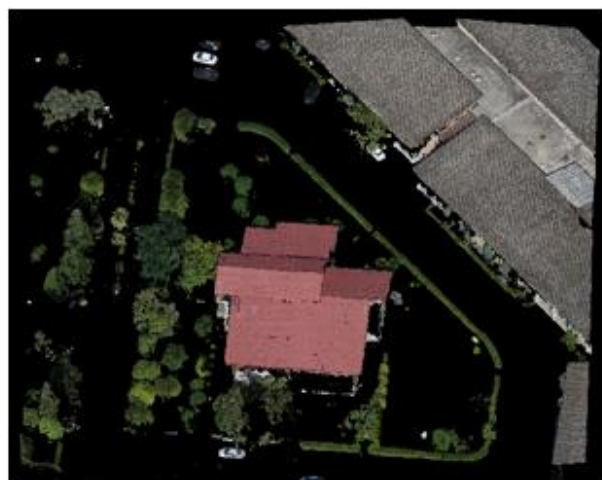

Pointcloud after ground filtering

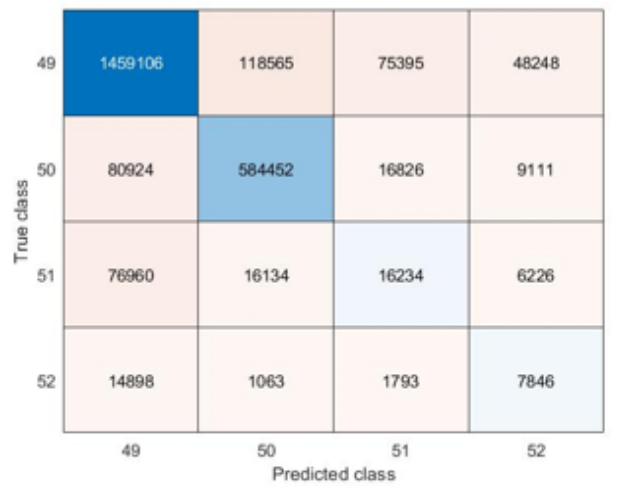

Confusion matrix

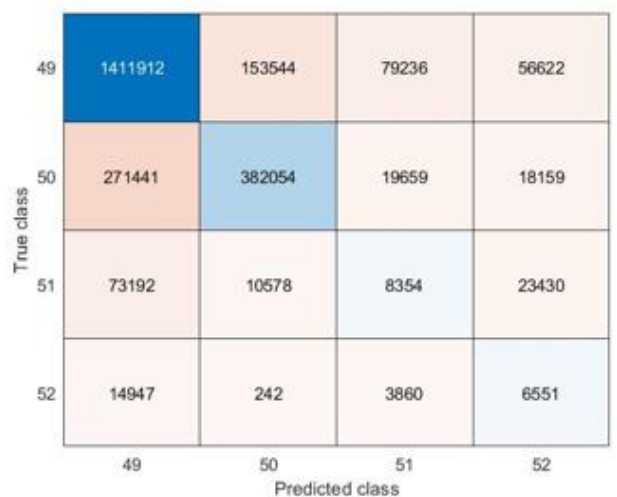

Confusion matrix

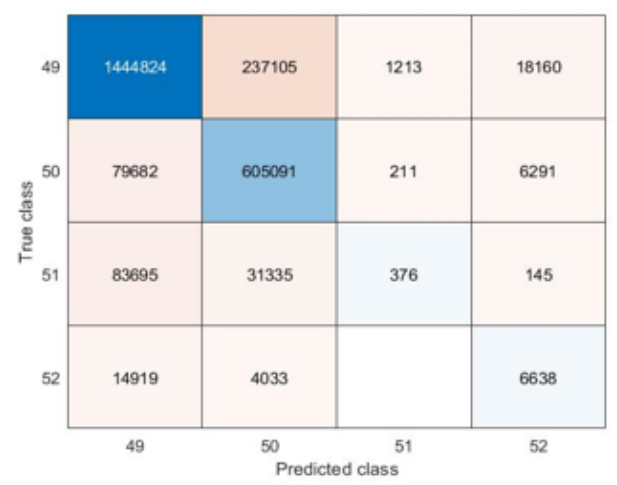

Confusion matrix

Figure 7. Classification results based different conditions 


\begin{tabular}{|c|c|c|c|c|c|}
\hline Features kind & Neighborhood & Specificity & Sensitivity & Prevalence & Overall accuracy \\
\hline $\begin{array}{l}\text { - Geometric } \\
\text { - Color }\end{array}$ & $\begin{array}{r}\square \text { segment-base } \\
\square \text { point-base }\end{array}$ & 0.7924 & 0.8567 & 0.6715 & 0.8152 \\
\hline $\begin{array}{l}\text { Geometric } \\
\text { - Color }\end{array}$ & $\begin{array}{r}\text { segment-base } \\
\text { a point-base }\end{array}$ & 0.5659 & 0.8303 & 0.6715 & 0.7434 \\
\hline $\begin{array}{c}\text { - Geometric } \\
\square \quad \text { Color }\end{array}$ & $\begin{array}{r}\text { - segment-base } \\
\square \text { point-base }\end{array}$ & 0.7887 & 0.8609 & 0.6715 & 0.8372 \\
\hline
\end{tabular}

Table 2. Evaluation of classification performance (in \%)

As Table 2, Sensitivity, specificity and overall accuracy are used for classification evaluation. Sensitivity, specificity and accuracy are described in terms of TP, TN, FN and FP.

Sensitivity $=\mathrm{TP} /(\mathrm{TP}+\mathrm{FN})=($ Number of true positive assessment)/ (Number of all positive assessment).

Specificity $=\mathrm{TN} /(\mathrm{TN}+\mathrm{FP})=($ Number of true negative assessment)/ (Number of all negative assessment).

Overall Accuracy $=(\mathrm{TN}+\mathrm{TP}) /(\mathrm{TN}+\mathrm{TP}+\mathrm{FN}+\mathrm{FP})=($ Number of correct assessments)/Number of all assessments).

\section{CONCLUSION}

In this research point-based and segment-based point cloud classification methods and using different kind of features were compared. Both point-based and segment-based methods have its pros and cons. Segment-based results have homogenous regions with lower noise, computational time and overall accuracy of $83.72 \%$ but it has some problems for edge points. Point-based method has better performance for edge points however it has more computational time and noise. Results shows that color features improve classification performance and it has an important role for vegetation and trees in classification. In future the role of different classifiers and use of more features will be investigated.

\section{REFRENCES}

Bremer, M., Wichmann, V. and Rutzinger, M., 2013. Eigenvalue and graph-based object extraction from mobile laser scanning point clouds. ISPRS Annals of the Photogrammetry, Remote Sensing and Spatial Information Sciences, 5, p.W2
Breiman, L, 2001. Random forests. Mach. Learn., 45, 5-32.

Chehata, N.; Guo, L.; Mallet, C, 2009. Airborne LiDAR feature selection for urban classification using random Forests. Int. Arch. Photogramm. Remote Sens.

Hackel, T.; Wegner, J.D.; Schindler, K, 2017. Joint classification and contour extraction of large 3D point clouds.ISPRS J. Photogramm. Remote Sens.

Luo, H.; Wang, C.; Wen, C.; Chen, Z.; Zai, D.; Yu, Y.; Li, J, 2018. Semantic Labeling of Mobile LiDAR Point CloudsVia Active Learning and Higher Order MRF. IEEE Trans. Geosci. Remote Sens.

Quan Li * and Xiaojun Cheng, 2018. Comparison of Different Feature Sets for TLS PointnCloud Classification.

Sun, Z.; Xu, Y.; Hoegner, L.; Stilla, U, 2018. Classification of Mls Point Clouds in Urban Scenes Using Detrended Geometric Features from Supervoxel-Based Local Contexts. ISPRS Ann. Photogramm. Remote Sens. Spat. Inf. Sci. 2018, 4, 271-278.

W. Zhang, J. Qi, P. Wan, H. Wang, D. Xie, X. Wang, and G. Yan, 2016. An Easy-to-Use Airborne LiDAR Data Filtering Method Based on Cloth Simulation, Remote Sens., vol. 8, no. 6, p. 501,

Yang, B.; Dong, Z.; Liu, Y.; Liang, F.;Wang, Y, 2017. Computing multiple aggregation levels and contextual features for road facilities recognition using mobile laser scanning data. ISPRS J. Photogramm. Remote Sens. 2017, 126,180-194. 